\title{
The Message for World Kidney Day 2009
}

\author{
Hypertension and Kidney Disease: A Marriage That Should Be Prevented
}

\author{
George L. Bakris ${ }^{a} \quad$ Eberhard Ritz ${ }^{b}$ \\ on behalf of the World Kidney Day Steering Committee \\ ${ }^{a}$ Department of Medicine, Hypertensive Diseases Unit, University of Chicago, Pritzker School of Medicine, \\ Chicago, III., USA; 'b Department of Medicine, Ruperto Carola University of Heidelberg, Heidelberg, Germany
}

\section{Introduction}

The kidney is both a cause and victim of hypertension. High blood pressure is a key pathogenetic factor that contributes to deterioration of kidney function. Presence of kidney disease is a common and underappreciated preexisting medical cause of resistant hypertension [1]. Therefore, treatment of hypertension has become the most important intervention in the management of all forms of chronic kidney disease (CKD). For this reason, the forthcoming World Kidney Day (WKD) on March 12th 2009 will emphasize the role of hypertension for renal disease.

\section{How Does One Recognize the Presence of Chronic Kidney Disease?}

In contrast to a decade ago, today most laboratories around the world report estimated glomerular filtration rate (eGFR) instead of or in addition to serum creatinine. This now provides the physician with information about kidney function that is, in general, more informative. As a result, a greater percentage of patients with diabetes or hypertension and their physicians have a better knowledge of their kidney function. Assessment of eGFR as an index of kidney function should be complemented by assessing urine for protein or albumin (preferred).
In spite of these laboratory updates, recent data demonstrate that a given patient's knowledge that he or she has CKD is very low. In a recent analysis of almost half a million people in Taiwan who took part in a standard medical screening program, 12 percent had CKD [2]. It was noteworthy that less than four percent of those with $\mathrm{CKD}$ were aware of their condition. People with CKD are several times more likely to die from cardiovascular (CV) causes than those without CKD, thus, hypertension is a major risk factor in this context [3]. The combination of CKD and hypertension, therefore, is a major public health issue; because of the costly treatments necessary for endstage renal disease (ESRD), end stage CKD has also become a substantial burden to health budgets.

\section{What is the Worldwide Frequency of Chronic Kidney Disease?}

The frequency of CKD continues to increase worldwide as does the prevalence of end-stage renal disease (ESRD) $[4,5]$. The most common, but not only, causes of

World Kidney Day 2009 Steering Committee: William Couser, Paul Beerkens, co-Chairmen. Tom Reiser, Jan Lantink, Project Directors. Georgi Abraham, Alan Collins, John Feehally, Joel Kopple, Philip Li, Miguel Riella, Bernardo Rodriguez-Iturbe, Anne Wilson.

Correspondence to: angela@worldkidneyday.org

\section{KARGER}

Fax +41613061234 E-Mail karger@karger.ch www.karger.com

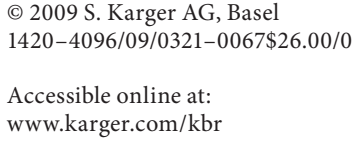

The University of Chicago, Pritzker School of Medicine

Hypertension Center, Section of Endocrinology, Diabetes and Metabolism

5841 S. Maryland Ave., MC 1027, Chicago, IL 60637 (USA)

Tel. + 1773702 7936, Fax +1 773834 0486, E-Mail gbakris@medicine.bsd.uchicago.edu 
CKD are hypertension and diabetes. The presence of CKD is associated with a large increase in cardiovascular (CV) risk. Moreover, CV risk increases proportionally as eGFR falls below $60 \mathrm{ml} / \mathrm{min}$. Lastly, death from CV causes is higher in CKD and much higher than is cancer in CKD; as a result the identification and reduction of CKD has become a public health priority [6].

The reported prevalence of CKD stages 1-4 in the most recent NHANES (national health and nutrition examination survey) between 1999 and 2006 was 26 million out of a population base of approximately 200 million. This represented United States residents aged 20 and older adult; of these, $65.3 \%$ had CKD stage 3 or 4 . Those with diabetes and hypertension had far greater prevalence of CKD (37 and 26\%, respectively) compared to those without these conditions (11 and 8\%, respectively) [7]. In a more recent, yet unpublished report from the CKD surveillance group, the prevalence or amount of CKD stages 1-4 in the general population increased 30\%, from 1994 to 2006 .

The most recent report of the United States Renal Data System estimates that nearly one-half million patients in the United States were treated for ESRD in the year 2004 [8], and by 2010 this figure is expected to increase by approximately $40 \%$. Patients destined to progress to ESRD, i.e. the elderly, are a growing segment of the population. Additionally, males and African-Americans with pre-existing hypertension and CKD are also at much higher risk for ESRD [9]. This observation has also been confirmed throughout the developed world: Europe, Asia, Australia and regions of India and Africa $[4,5]$.

\section{The Role of Hypertension}

Hypertension is a global problem, and the situation is projected to get worse. It is the major risk factor for development and progression in nondiabetic and diabetic CKD.

The world population is getting older and aging is the most common risk factor for the development of hypertension and diabetes as well as CKD. Nearly one billion people worldwide have high blood pressure (defined as $>140 / 90 \mathrm{~mm} \mathrm{Hg}$ ), and that number is expected to increase to 1.56 billion people by 2025 [10]. The prevalence of hypertension is predicted to increase by 24 percent in developed countries and by 80 percent in developing regions such as Africa and Latin America. One report noted that 333 million adults in economically developed regions such as North America and Europe had high blood pressure in 2000, and an additional 639 million people in developing countries have this condition.

In 1999-2006, the prevalence of hypertension in US adults was $43.4 \%$ when defined as $>140 / 90 \mathrm{~mm} \mathrm{Hg}$ and similar figures have been reported from many Western countries [9]. The rates of hypertension were highest in participants who were 60 years or older, i.e. $68-80 \%$ versus $25 \%$ in those between 20-to 39-years, in non-Hispanic blacks (53\%) versus Caucasians (43\% vs. MexicanAmericans (34\%). Furthermore, hypertension was more common in individuals with a higher body mass index (BMI) (60\% for BMI $\geq 35$ vs. $32 \%$ for BMI of 23 ). Slightly more than half of adults with hypertension were aware of their disease in 1999-2004; fewer than half were treated for their hypertension with medications; and less than two-thirds were controlled to $<140 / 90 \mathrm{~mm} \mathrm{Hg}$ with medication [9]. This trend in poor blood pressure control is observed worldwide.

The hypertension control rate is substantially less in patients with CKD particularly those with diabetes and CKD $[1,9]$. This is illustrated by the National Kidney Foundation's(USA) Kidney Early Evaluation Program (KEEP), a US-based health-screening program for individuals at high risk for kidney disease [9]. The prevalence (86.2\%), awareness $(80.2 \%$,), and treatment $(70.0 \%)$ of hypertension in the screened cohort were high, however, blood pressure control rates were low (13.2\%). The proportion of hypertensive patients increased with advancing stages of CKD.

\section{Which Blood Pressure Component is Most Relevant for Renal and Cardiovascular Risk, Systolic or Diastolic?}

There is now consensus, based on the totality of the data, that systolic rather than diastolic blood pressure poses the greater risk for cardiovascular events and kidney disease progression. Against this background, it is relevant that in the KEEP study elevated systolic blood pressure accounted for the majority of patients with inadequate control. Male gender, non-Hispanic black race, and BMI of $30 \mathrm{~kg} / \mathrm{m}^{2}$ or more were inversely related to blood pressure control.

What is the blood pressure target for CKD patients? According to the different guidelines published by the major kidney societies, systolic blood pressure should be lowered to values $<130 \mathrm{~mm} \mathrm{Hg}$. One has to be aware, however, that as a predictor of adverse CKD or cardiovascular events, office blood pressure may be inferior com- 
pared to ambulatory blood pressure measurement [11]. This issue is particularly relevant in CKD because of the tendency for nighttime blood pressure to be elevated (little or no nocturnal dip in blood pressure) and the fact that central (aortic) blood pressure tends to be higher than peripheral (brachial) blood pressure [11, 12]. In patients with diabetes, guidelines all recommend that lower blood pressure targets may provide further benefit, but prospective trials have thus far, failed to confirm this epidemiological observation.

\section{The Role of Diabetic Nephropathy}

As indicated above, diabetes and hypertension are the most common causes of CKD. There are currently over 240 million people with diabetes worldwide. This figure is projected to rise to 380 million by 2025 , largely due to population growth, aging, urbanization, unhealthy eating habits, increased body fat and a sedentary lifestyle. By 2025 ,the number of people with diabetes is expected to more than double in South-East Asia, the Eastern Mediterranean and Middle East, and Africa. It is projected to rise by nearly 20\% in Europe, 50\% in North America, $85 \%$ in South and Central America and 75\% in the Western Pacific region. The top five countries with the highest prevalence of diabetes in order include India, China, the United States, Russia and Japan. Worldwide more than $50 \%$ of people with diabetes are unaware of their condition and are not treated.

The same behaviors that increase obesity are shared with those predisposed to diabetes, i.e. family history, presence of hypertension, ageing, excess body weight, lack of exercise and unhealthy dietary habits. It is important to identify these risks early to reduce the development of diabetes and CKD, since CKD greatly amplifies the risk of cardiovascular events in the diabetic patient.

\section{The Remaining Challenge}

Under diagnosis and under treatment of CKD is a worldwide problem: not only is CKD awareness low worldwide, but the relative lack of CKD risk factor awareness by physicians i.e. hypertension and diabetes is even more disturbing. Moreover, even awareness of these risk factors does not ensure adequate treatment; this could relate either to the behavior of the patient, the provider or both. Thus, the problem of CKD remains a challenge as exemplified by recent data showing that between 1999-
$2006,<5 \%$ of people with an eGFR $<60 \mathrm{ml} / \mathrm{min} / 1.73 \mathrm{~m}^{2}$ and proteinuria were aware of having CKD; of those with CKD stage 3 , awareness was only $7.5 \%$; for stage 4 , awareness was less than 50\%. Awareness rates among those with CKD stages 3 or 4 were higher if co-morbid diagnoses of diabetes and hypertension were present, but even then, they were quite low (20 and $12 \%$, respectively).

One barrier to overcome in order to ensure greater awareness is a more focused education of physicians, since they are the purveyors of the patients' medical condition. In one survey, more than one-third of primary care physicians in the US were not aware that family history was a risk factor for $\mathrm{CKD}$, while almost one-quarter did not perceive African-American ethnicity as a CKD risk factor; in contrast, nearly all perceived diabetes (95\%)and hypertension (97\%) as risk factors for CKD. Even more problematic was the fact that while diabetes and hypertension were acknowledged as CKD risk factors the achieved control rates (defined as reaching guideline goals) sadly remains well below 50\% among those treated.

\section{What Can Be Done about This Problem?}

There have been many consensus panels over the past decade to approach ways to achieve better blood pressure control and educate physicians to the stages of CKD [13, 14].The road to improve outcomes is to focus on public awareness and screening programs as well as programs to educate both patients and physicians. Data from the KEEP screening program in the US has also noted that blood pressure values are most likely to be at goal once a patient is aware they have kidney disease [15]. Data from Bolivia highlights the observation that once kidney disease is diagnosed more appropriate interventions to reduce CKD risk factors such as hypertension are instituted [13].

Programs to address these issues have started around the world including KEEP type programs. As a major focus of World Kidney Day this year the issue is hypertension in CKD (http: //www.worldkidneyday.org)

Because of the aging world population and consequent increasing prevalence of hypertension and diabetes, CKD rates will continue to increase. This has and will continue to place an undue economic burden on societies given the costs for an ESRD program. In 2005, the US spent USD 32 billion dollars on such programs. These facts mandate that measures be put forth to ensure timely detection and prevention of CKD progression. The key to ensure suc- 
cessful prevention of CKD is screening for hypertension, improved testing and diagnosis of predisposing co-morbidities such as diabetes and aggressive treatment to guideline goals.

The International Society of Nephrology (ISN) and the International Federation of Kidney Foundations (IFKF) have an ambitious long-term goal that worldwide, that every individual, particularly the patient with diabetes, knows his or her blood pressure values. Additionally, they should be aware that prompt treatment is necessary once blood pressure values are no longer in the normal range. Finally our societies strongly encourage public health authorities to support efforts to raise public awareness of CKD and promote moves to reduce the risk of developing hypertension. Such governmental public health initiatives are exemplified by countries like the United Kingdom, Finland and Japan reducing salt in the diet and mandating labels have sodium content as in the US. These initiatives have proven highly successful based on reduction in cardiovascular mortality and morbidity.

\section{References}

1 Sarafidis PA, Bakris GL: State of hypertension management in the United States: confluence of risk factors and the prevalence of resistant hypertension. J Clin Hypertens (Greenwich) 2008;10:130-139.

$\checkmark 2$ Wen CP, Cheng TY, Tsai MK, et al: All-cause mortality attributable to chronic kidney disease: a prospective cohort study based on 462 293 adults in Taiwan. Lancet 2008;371:21732182.

3 McCullough PA, Jurkovitz CT, Pergola PE, et al: Independent components of chronic kidney disease as a cardiovascular risk state: results from the Kidney Early Evaluation Program (KEEP). Arch Intern Med 2007;167: 1122-1129.

$\checkmark 4$ Atkins RC: The epidemiology of chronic kidney disease. Kidney Int Suppl 2005;94: S14-S18.

5 Alebiosu CO, Ayodele OE: The global burden of chronic kidney disease and the way forward. Ethn Dis 2005; 15:418-423.
-6 Rosamond W, Flegal K, Furie K, et al: Heart disease and stroke statistics - 2008 update: a report from the American Heart Association Statistics Committee and Stroke Statistics Subcommittee. Circulation 2008;117:e25e146.

7 Ostchega Y, Yoon SS, Hughes J, Louis T: Hypertension Awareness, Treatment, and Control -Continued Disparities in Adults: United States, 2005-2006. NCHS Data Brief www.cdc.gov/nchs/data/databriefs/db03. pdf, 2008.

8 Coresh J, Selvin E, Stevens LA, et al: Prevalence of chronic kidney disease in the United States. JAMA 2007;298:2038-2047.

-9 Sarafidis PA, Li S, Chen SC, et al: Hypertension awareness, treatment, and control in chronic kidney disease. Am J Med 2008;121: 332-340.

10 Kearney PM, Whelton M, Reynolds K, et al: Global burden of hypertension: analysis of worldwide data. Lancet 2006;365:217-223.
11 Peterson GE, de Backer T, Gabriel A, et al: Prevalence and correlates of left ventricular hypertrophy in the African American Study of Kidney Disease Cohort Study. Hypertension 2007;50:1033-1039.

12 Townsend RR: Analyzing the radial pulse waveform: narrowing the gap between blood pressure and outcomes. Curr Opin Nephrol Hypertens 2007;16:261-266.

13 Perico N, Plata R, Anabaya A, et al: Strategies for national health care systems in emerging countries: the case of screening and prevention of renal disease progression in Bolivia. Kidney Int Suppl 2005;S87-S94.

14 Whelton PK, Beevers DG, Sonkodi S: Strategies for improvement of awareness, treatment and control of hypertension: results of a panel discussion. J Hum Hypertens 2004; 18:563-565.

15 Rao MV, Qiu Y, Wang C, Bakris G: Hypertension and CKD: Kidney Earl Evaluation Program (KEEP) and National Health and Nutrition Examination Survey (NHANES), 1999-2004. Am J Kidney Dis 2008;51:S30S37. 